\title{
Management Decisions in Nontraumatic Complaints of Arm, Neck, and Shoulder in General Practice
}

Anita Feleus, $P b D^{1,2}$

Sita M. A. Bierma-Zeinstra, $P b D^{1}$

Roos M. D. Bernsen, $P b D^{3}$

Harald S. Miedema, $M D^{2}$

Jan A. N. Verbaar, $P b D^{4}$

Bart W. Koes, $P b D^{1}$

'Department of General Practice, Erasmus Medical Centre, Rotterdam, The Netherlands

${ }^{2}$ Netherlands Expert Center for WorkRelated Musculoskeletal Disorders, Erasmus Medical Centre, Rotterdam, The Netherlands

${ }^{3}$ Department of Community Medicine, Faculty of Medicine and Health Sciences, United Arab Emirates University, Al Ain, United Arab Emirates

${ }^{4}$ Department of Orthopaedics, Erasmus Medical Centre, Rotterdam, The Netherlands

Conflicts of interest: none reported.

\section{CORRESPONDING AUTHOR}

Anita Feleus, $\mathrm{PhD}$

Department of General Practice, Room Wk121

Erasmus Medical Center Rotterdam PO Box 2040

3000 CA Rotterdam, The Netherlands a.feleus@erasmusmc.nl

\begin{abstract}
OBJECTIVE We wanted to evaluate associations between diagnosis and characteristics of the patient, complaint, and general practitioner (GP), as well as 6 common management decisions, in patients with nontraumatic arm, neck, and shoulder complaints at the time of the first consultation with their physician.

METHODS We undertook an observational cohort study set in 21 Dutch general practices, including 682 patients with nontraumatic complaints of arm, neck, and shoulder. The outcome measure was application (yes/no) of a specific management option: watchful waiting, additional diagnostic tests, prescription of medication, corticosteroid injection, referral for physiotherapy, and referral for medical specialist care.

RESULTS Separate multilevel analyses showed that overall, the diagnostic category, having long duration of complaints, and reporting many functional limitations were most frequently associated with the choice of a management option. For watchful waiting, only complaint variables played a role (long duration of complaints, high complaint severity, many functional limitations, recurrent complaint). All these variables were negatively associated with watchful waiting. When opting for 1 of the 5 other management options, several physician characteristics played a role as well. Less clinical experience was associated with additional diagnostic tests and referral to a medical specialist. GPs working in a solo practice more frequently referred to a medical specialist. GPs working in a rural area more frequently referred for physiotherapy. Female GPs prescribed medication less frequently. Physicians with special interest in musculoskeletal complaints gave corticosteroid injections more frequently.
\end{abstract}

CONCLUSIONS Diagnostic category, long duration of complaints, and high functional limitations were key variables in management decisions with these complaints. In addition, several physician characteristics played a role as well.

Ann Fam Med 2009;7:446-454. doi:10.1370/afm.993.

\section{INTRODUCTION}

omplaints of arm, neck, and shoulder are very common in Western societies. ${ }^{1,2}$ In the Netherlands the estimated 12 -month prevalence

in the general population was $31 \%$ for neck pain, $30 \%$ for shoulder pain, $11 \%$ for elbow pain, and $18 \%$ for wrist or hand pain. ${ }^{1,2}$ Studies have reported that of the respondents with noninflammatory musculoskeletal pain, about $30 \%$ to $45 \%$ contacted their general practitioner (GP). ${ }^{1,3}$ In Dutch general practice, incidence data for patients with nontraumatic arm, neck, or shoulder complaints show 97 consultations per 1,000 registered persons annually. ${ }^{4}$

Common management options for patients with nontraumatic arm, neck, and shoulder complaints are watchful waiting, additional diagnostic tests, prescription of medication, referral for physiotherapy, a corticosteroid injec- 
tion, and referral for medical specialist care. Use of these 6 management options shows wide variation, however, both between and within diagnostic groups. ${ }^{5}$ Until now, no studies have evaluated the determinants that contribute to variation in the management of these complaints. Part of this variation may be explained by the diagnosis, which, because of its natural course and available treatment, usually guides management. Also, patient and complaint characteristics may influence management. In the Netherlands guidelines issued by the Dutch College of General Practitioners are available for epicondylitis ${ }^{6}$ and shoulder complaints ${ }^{7}$; in both guidelines, management advice is partly based on differences in the levels of hindrance (pain severity and functional limitations). In other study populations, patient and complaint characteristics reported to be associated with management options are distress, poor perceived health, age, and sex. ${ }^{8-10}$ Additionally, indicators of poor prognosis can play a role in management decisions. In our earlier study in this population, indicators of poor prognosis were long duration of the complaints at baseline, having musculoskeletal comorbidity, recurrent complaint, low social support, and a high somatization level. ${ }^{11}$

Although GP characteristics, such as sex, years of experience, and type of practice, are also reported to influence management decisions, ${ }^{12,13}$ in a well-organized health care system, their influence would be expected to be marginal and that the diagnosis, patient, and complaint characteristics would show the strongest associations with management. Furthermore, highly variable treatment that cannot be explained by differences in diagnoses and patient or complaint characteristics could indicate the need for treatment guidelines to ensure that management decisions at least can be based on available knowledge on preferable outcomes or, when not available, on costs.

This study aimed to evaluate the association of the diagnosis, characteristics of the patient, complaint, and GP with the 6 common management decisions in patients with nontraumatic arm, neck, and shoulder complaints at the time of the first consultation with their GP.

\section{METHODS}

The present study is part of a larger cohort study performed in the southwestern region of the Netherlands involving 21 general practices. The Medical Ethics Committee of the Erasmus Medical Center approved the study protocol.

\section{Study Population}

In total, 31 GPs recruited eligible patients from September 2001 through December 2002. Included were patients aged 18 to 64 years who consulted their GP for a new complaint or a new episode of complaints of neck, upper back, shoulder, upper arm, elbow, forearm, wrist, or hand. The episode was considered new if patients had not consulted their GP for the same complaint during the preceding 6 months. Excluded were patients for whom the complaint could be explained by a trauma, fracture, malignancy, amputation, prosthesis, congenital defect, or previously diagnosed systemic or generalized neurological disorder.

\section{Procedures}

Before the GPs started to recruit patients, the GP characteristics were gathered during a structured interview.

During the first consultation patients received study information, an informed consent form, and a selfadministered questionnaire from their GP. A fax was then sent to the research team with the initial diagnosis, expected prognosis at 6 months, and whether the complaint was recurrent. After the research team had received the completed informed consent and questionnaire (within 8 weeks), inclusion criteria were verified in the electronic medical file. Data on treatment management at the time of the consultation and patient and complaint characteristics were extracted from the selfadministered questionnaire.

\section{Outcome}

Data on the following 6 common management options (yes/no) were gathered: request additional diagnostic tests (in addition to history taking and physical examination), give a corticosteroid injection, prescribe a medication (injections excluded), refer to a physiotherapist, refer to a medical specialist, and watchful waiting.

\section{Variables Possibly Associated With Management}

Possible associated variables were assigned to 1 of 3 domains: (1) diagnosis, (2) patient and complaint characteristics, and (3) GP characteristics. In accordance with the Dutch guidelines for shoulder complaints and epicondylitis, the diagnoses were categorized into 4 groups: specific shoulder diagnosis (impingement, frozen shoulder, biceps tendonitis), epicondylitis, other specific diagnosis, and nonspecific diagnosis. ${ }^{6,7,14}$

Patient and complaint characteristics included age and sex, having employment, complaint severity, ${ }_{1}^{15}$ functional limitations, ${ }^{16}$ perceived general health, ${ }^{17}$ musculoskeletal comorbidity, ${ }_{1}^{1,3}$ recurrent vs incident complaint, multiple-region complaint, social support, ${ }^{18}$ and somatization and distress. ${ }^{19} \mathrm{GP}$ characteristics included sex, years in practice, special interest in musculoskeletal complaints, continuing education in musculoskeletal complaints, whether the physician was in group practice, and practice location (rural vs urban). ${ }^{20}$

In addition, at the first consultation the treating 
GP scored expected nonrecovery at 6 months on a 4 point Likert scale ranging from very likely (1) to very unlikely (4). These scores were dichotomized into likely or unlikely.

\section{Patient/Complaint Characteristics}

Table 1 shows the distribution of the patient and complaint characteristics of the 682 patients in the current study. Of the total study population $47 \%$ was male

\section{Statistical Analyses}

We evaluated the independent relationship of the variables of the 3 domains (diagnosis, patient and complaint characteristics, and GP characteristics) with the 6 management options by means of logistic regression for repeated measurements (generalized estimating equations), ${ }^{21,22}$ with physicians as units of analysis and compound symmetry as variance/covariance structure. This model takes into account the correlation between patients consulting the same physician (PROC GENMOD, SAS version 8.2, SAS Institute, Cary, North Carolina).

Separate models were constructed for the 6 management options. For possible associated factors with clinically relevant classifications or validated classes, the existing cutoff points were used. If cutoff points were not available, ordinal scores were split based on the median score of the total population.

Analyses were performed both in univariate and multivariate models, resulting in the assessment of odds ratios (ORs) and corresponding 95\% confidence intervals (CIs). First, variables with $P<.20$ in the univariate model were selected for multivariate analysis (enter procedure) within the domains of patient and complaint characteristics and GP characteristics separately. Second, variables with $P<.20$ in the multivariate model per domain were selected for the final analyses. Finally, variables with a significant odds ratio $(P<.05)$ were kept in the multivariate model and presented in the results. The diagnostic group variable entered the multivariate model and was kept in the multivariate model independent of the level of significance. The largest diagnostic group is defined as the reference group because doing so is statistically the most efficient.

\section{RESULTS}

\section{Response}

In total, 798 patients fulfilled the entry criteria, and 682 (86\%) entered the cohort after they returned the completed questionnaire and informed consent. The mean time between consultation and filling in the questionnaire was 2 weeks.

\begin{tabular}{|c|c|}
\hline Variables & Value \\
\hline \multicolumn{2}{|l|}{ Diagnosis ( $n=682$ patients) ${ }^{6,7,14}$} \\
\hline Epicondylitis, n (\%) & $95(14)$ \\
\hline Impingement, biceps tendonitis, frozen shoulder, n (\%) & $229(34)$ \\
\hline Other specific diagnosis, $\mathrm{n}(\%)$ & $78(11)$ \\
\hline Nonspecific diagnosis, n (\%) & $280(41)$ \\
\hline \multicolumn{2}{|l|}{ Patient and complaint characteristics ( $n=682$ patients) } \\
\hline Age, $y$, median (range) ${ }^{a}$ & $45(18-64)$ \\
\hline Female, n (\%) & $399(58)$ \\
\hline Not having paid work, $\mathrm{n}(\%)$ & $148(22)$ \\
\hline \multicolumn{2}{|l|}{ Duration of the complaint at consultation } \\
\hline $0-6 w k, n(\%)$ & $344(51)$ \\
\hline 6 wk-6 mo, n (\%) & $162(24)$ \\
\hline$>6 \mathrm{mo}, \mathrm{n}(\%)$ & $175(25)$ \\
\hline Complaint severity in last week, median (range) ${ }^{\mathrm{a}, \mathrm{b}}$ & $6[1-10]$ \\
\hline Functional limitations, DASH, median (range) ${ }^{\mathrm{a}, \mathrm{c}}$ & $35.4(2.9-99.1)$ \\
\hline Poor perceived general health, SF-12 question No. 1, n (\%) ${ }^{d}$ & $86(13)$ \\
\hline Musculoskeletal comorbidity, n (\%) & 331 (49) \\
\hline Recurrent complaint vs incident complaint, $\mathrm{n}(\%)$ & $191(28)$ \\
\hline Multiple-region complaint, $n(\%)^{e}$ & $287(42)$ \\
\hline Low social support, SOS score $<56, n(\%)^{a, f}$ & $342(50)$ \\
\hline \multicolumn{2}{|l|}{ Somatization, 4DSQ, n (\%) ${ }^{g}$} \\
\hline Low (score 0-10) & $503(74)$ \\
\hline Medium (score 11-20) & $148(22)$ \\
\hline High (score 21-32) & $30(4)$ \\
\hline Distress, 4DSQ, n (\%) ${ }^{g}$ & $432(63)$ \\
\hline \multicolumn{2}{|l|}{ Low (score $0-10$ ) } \\
\hline Medium (score 11-20) & $170(25)$ \\
\hline High (score 21-32) & $79(12)$ \\
\hline \multicolumn{2}{|l|}{ GP characteristics $(n=31)$} \\
\hline Female, n (\%) & $5(16)$ \\
\hline Years of practice, median (range) & $13(1-35)$ \\
\hline Special interest in musculoskeletal complaints, n (\%) & $16(53)$ \\
\hline $\begin{array}{l}\text { Continuing medical education in musculoskeletal } \\
\text { complaints, } \mathrm{n}(\%)\end{array}$ & $13(41)$ \\
\hline \multicolumn{2}{|l|}{ GP practice characteristics $(n=21)$} \\
\hline Group practice & $11(52)$ \\
\hline Rural vs urban ${ }^{h}$ & $8(38)$ \\
\hline
\end{tabular}

$\mathrm{GP}=$ general practitioner; DASH = Disability of Arm, Shoulder and Hand questionnaire; $4 D S Q=$ Four-Dimensional Symptom Questionnaire; SF-12 = SF-12 Health Survey; SOS = Social Support Scale, a Dutch version of the Social Support Questionnaire.

a Dichotomous, with split based on median score of the total population.

b Scored on a range from 1 to 10 with 10 being the most severe, and 6 is the median.

c Each item scored on a 5-point Likert scale and summed and transferred to a score ranging from 0 (no disability) to 100 (completely disabled). ${ }^{15}$

${ }^{d}$ Four answer categories dichotomized as poor (fair or poor) vs good (excellent or very good). ${ }^{16}$

e Area with the most pain or complaints during the previous week indicated area on a mannequin: multiple regional vs regional. Defined regions are neck-shoulder, including neck; upper part of thoracic spine, shoulder and upper arm; elbow-forearm; and wrist-hand. ${ }^{17}$

f Scored on a range from 12 to 60 ; a higher score indicates more support. ${ }^{18}$

9 Scored on a range from 0 to 32; a higher score indicates more somatization or distress. ${ }^{19}$

${ }^{\mathrm{h}}$ Urbanization rate: rural $=$ addresses $\leq 1,000 / \mathrm{km}^{2}$, urban $=$ addresses $>1,000 / \mathrm{km}^{2} .{ }^{20}$ 
Table 2. Management of Nontraumatic Arm, Neck, and Shoulder Complaints

\begin{tabular}{|c|c|c|c|c|c|}
\hline Management Option & $\begin{array}{c}\text { Total } \\
\text { Population } \\
\text { (n = 682) } \\
n(\%)\end{array}$ & $\begin{array}{c}\text { Epicondylitis } \\
(n=95) \\
n(\%)\end{array}$ & $\begin{array}{l}\text { Impingement, } \\
\text { Biceps Tendonitis, } \\
\text { Frozen Shoulder } \\
(n=229) \\
n(\%)\end{array}$ & $\begin{array}{c}\text { Other Specific } \\
\text { Diagnosis } \\
(\mathrm{n}=78) \\
\mathrm{n}(\%)\end{array}$ & $\begin{array}{c}\text { Nonspecific } \\
\text { Diagnosis } \\
(\mathrm{n}=280) \\
\mathrm{n}(\%)\end{array}$ \\
\hline Additional diagnostic tests & $59(9)$ & $3(3)$ & $13(6)$ & $13(17)$ & $30(11)$ \\
\hline Corticosteroid injection & $71(11)$ & $5(5)$ & $50(22)$ & $9(12)$ & $7(3)$ \\
\hline Prescribe a medication & $235(35)$ & $31(33)$ & $97(42)$ & $23(30)$ & $84(30)$ \\
\hline Refer to physiotherapy & $158(23)$ & $24(25)$ & $40(18)$ & $9(12)$ & $85(30)$ \\
\hline Refer to medical specialist & $31(5)$ & $3(3)$ & $12(5)$ & $9(12)$ & $7(3)$ \\
\hline Watchful waiting & $276(41)$ & $46(48)$ & $74(32)$ & $36(46)$ & $120(43)$ \\
\hline
\end{tabular}

and the median age was 45 years. One-half (51\%) of the participants consulted their GP within 6 weeks after developing the complaint. After consultation, 229 patients $(34 \%)$ received a specific shoulder diagnosis (impingement, biceps tendonitis, frozen shoulder), 95 (14\%) received a diagnosis of epicondylitis, and 78 (11\%) received another specific diagnosis (eg, carpal tunnel syndrome, de Quervain tenosynovitis, etc); in 280 patients $(41 \%)$ no specific diagnosis was registered (Supplemental Appendix 1, available online at http:// annfammed.org/cgi/content/full/7/5/446/DC1).

Hir Complaint severity in the previous week showed a median score of 6 (range 1-10) and functional limitations of arm, neck, and shoulder showed a median score of 35.4 (range 2.9-99.1).

\section{Physician Characteristics}

Of the 31 recruiting GPs, 5 (16\%) were female. The reported years of practice as a GP ranged from 1 to 35 years (median 13) years; $53 \%$ of the physicians reported to have a special interest in musculoskeletal complaints. About one-half of the practices were solo practices, and the remainder were group practices.

\section{Management}

Table 2 displays the treatment options applied in both the total population and by diagnostic group. Within a maximum of 8 weeks, $9 \%$ of the total population received additional diagnostic testing, in $11 \%$ corticosteroid injections were given, and in 35\% medication was prescribed. Of all patients, $23 \%$ was referred for physiotherapy treatment and $5 \%$ for medical specialist consultation. In $41 \%$ of the patients none of the specified options was applied.

\section{Management Decisions}

The complaint characteristics of long duration of complaints, high complaint severity, high functional limitations, and having a recurrent complaint were all negatively associated with watchful waiting (Table 3 ).
Variables positively associated with the application of additional diagnostic tests were longer duration of complaints, a multiple-region complaint, and the GP having less than 13 years of practice (Table 3). Negatively associated variables were having a diagnosis of epicondylitis (compared with a nonspecific diagnosis) and the GP working in a group practice.

Variables positively associated with a prescription of a medication were having a specific shoulder diagnosis, high functional limitations, high somatization, short duration of complaints, and the GP being male (Table 3).

Variables positively associated with application of corticosteroid injections were having a specific shoulder diagnosis or other specific diagnosis, consulting a GP with special interest in musculoskeletal complaints, longer duration of complaints, high functional limitations, and being older (Table 4).

Variables positively associated with referral for physiotherapy were longer duration of complaints, having a recurrent complaint, having more distress, and consulting a GP in a rural area (Table 4).

Having a specific shoulder diagnosis or another specific diagnosis was negatively associated with referral for physiotherapy, but it was strongly positively associated with referral to a medical specialist. Furthermore, patients who reported higher functional limitations or consulted a GP with fewer years of experience were more frequently referred to a medical specialist.

In addition to the diagnostic category, a longer duration of complaints and higher functional limitations proved to be the patient and complaint variables most frequently associated with the 6 evaluated options in initial management of patients with nontraumatic arm, neck, and shoulder complaints (Table 5).

In watchful waiting, only long duration of complaints, high complaint severity, many functional limitations, and a recurrent complaint played a role. In patients with these complaint variables, watchful waiting was opted for less frequently. 


\begin{tabular}{|c|c|c|c|}
\hline Variables & $\begin{array}{l}\text { Watchful } \\
\text { Waiting } \\
\text { OR }(95 \% \mathrm{Cl})\end{array}$ & $\begin{array}{c}\text { Additional } \\
\text { Diagnostic Testing } \\
\text { OR }(95 \% \mathrm{Cl})\end{array}$ & $\begin{array}{l}\text { Prescription } \\
\text { of Medication } \\
\text { OR }(95 \% \mathrm{Cl})\end{array}$ \\
\hline \multicolumn{4}{|l|}{ Diagnosis } \\
\hline Epicondylitis & $1.2(0.8-1.7)$ & $0.2(0.1-0.5)$ & $1.2(0.8-1.6)$ \\
\hline Impingement, biceps tendonitis, frozen shoulder & $0.6(0.4-1.2)$ & $0.5(0.2-1.2)$ & $1.7(1.2-2.5)$ \\
\hline Other specific diagnosis & $1.1(0.7-1.7)$ & $1.3(0.9-2.0)$ & $1.1(0.7-1.8)$ \\
\hline Nonspecific diagnosis, ref & 1.0 & 1.0 & 1.0 \\
\hline \multicolumn{4}{|l|}{ Patient and complaint characteristics } \\
\hline \multicolumn{4}{|l|}{ Duration of the complaint at consultation } \\
\hline $0-6$ wk (ref) & 1.0 & 1.0 & 1.0 \\
\hline 6 wk-6 mo & $0.7(0.5-1.0)$ & $2.1(0.9-5.1)$ & $0.7(0.5-1.0)$ \\
\hline$>6 \mathrm{mo}$ & $0.6(0.4-0.8)$ & $5.0(2.4-10.5)$ & $0.6(0.4-0.9)$ \\
\hline High complaint severity in the last week (score $>6$ ) vs lowa & $0.6(0.4-0.9)$ & - & - \\
\hline Many functional limitations (DASH score $>35.34$ ) vs few ${ }^{b}$ & $0.6(0.5-0.9)$ & & $1.7(1.2-2.4)$ \\
\hline Recurrent complaint vs incident complaint & $0.7(0.6-0.9)$ & - & - \\
\hline Multiple-region complaint vs single region & - & $2.3(1.2-4.3)$ & \\
\hline Somatization, 4DSQc & - & - & - \\
\hline Low (score 0-10) & - & - & 1.0 \\
\hline Medium (score 11-20) & - & - & $1.0(0.7-1.5)$ \\
\hline High (score 21-32) & - & - & $2.5(1.2-5.2)$ \\
\hline \multicolumn{4}{|l|}{ GP characteristics } \\
\hline Female vs male & - & - & $0.6(0.4-0.9)$ \\
\hline Few years of practice ( $<13$ years) vs many & - & $2.9(1.8-4.7)$ & - \\
\hline Group practice vs solo practice & - & $0.5(0.3-0.9)$ & - \\
\hline \multicolumn{4}{|c|}{ Note: All variables in the final model have a multivariate association with a $P$ value $<.05$. } \\
\hline \multicolumn{4}{|c|}{$\begin{array}{l}\mathrm{Cl}=\text { confidence interval; } \mathrm{DASH}=\text { Disability of Arm Shoulder and Hand questionnaire; } 4 \mathrm{DSQ}=\text { Four-Dimensional Symptom Questionnaire; } \mathrm{OR}=\text { odds ratio; ref }=\text { refer- } \\
\text { ence group. }\end{array}$} \\
\hline \multicolumn{4}{|c|}{$\begin{array}{l}\text { a Scored on a range from } 1 \text { to } 10 \text { with } 10 \text { being the most severe, and } 6 \text { is the median. } \\
\text { b Each item scored on a } 5 \text {-point Likert scale and summed and transferred to a score ranging from } 0 \text { (no disability) to } 100 \text { (completely disabled). }{ }^{15} \\
\text { c Scored on a range from } 0 \text { to } 32 \text {; a higher score indicates more somatization or distress. }{ }^{19}\end{array}$} \\
\hline
\end{tabular}

The results of the univariate analysis are presented in Supplemental Appendix 2 (available online at http://annfammed.org/cgi/content/full/7/5/446/ DC1).

\section{DISCUSSION}

Salem-Schatz et al reported that patient and complaint characteristics are important in management decisions ${ }^{23}$; however, their indicators of referral were limited to data on age, sex, and severity of illness based on diagnostic codes from automated medical record systems. In the present study we were able to include a wider range of patient and complaint characteristics (eg, functional limitations, complaint severity, etc), and we expected the GP characteristics to play only a limited role.

In contrast to our expectations, however, when opting for 1 of the 5 management options other than watchful waiting, several GP characteristics did play a role as well.

\section{Influence of GP Characteristics}

As expected, GP characteristics were not associated with watchful waiting; only complaint characteristics played an independent role. Several GP characteristics did play an independent role in the other 5 management options, however. Working in a solo practice and less practice experience were associated with additional diagnostic tests. The latter variable was also associated with referral to a medical specialist. These GP characteristics may imply some uncertainty regarding the diagnosis. More referral to physical therapists made by GPs in rural areas may reflect a closer cooperation between caregivers in such areas. Furthermore, female GPs prescribed relatively less medication compared with their male colleagues, although they did not use any other option more frequently.

Corticosteroid injections were most frequently given by those GPs with a special interest in musculoskeletal complaints. Their interest might result in more knowledge and training regarding these complaints (including giving a corticosteroid injection) 


\begin{tabular}{|c|c|c|c|}
\hline Variables & $\begin{array}{l}\text { Steroid Injection } \\
\text { OR }(95 \% \mathrm{Cl})\end{array}$ & $\begin{array}{l}\text { Physical Therapist } \\
\text { OR }(95 \% \mathrm{Cl})\end{array}$ & $\begin{array}{l}\text { Medical Specialist } \\
\text { OR }(95 \% \mathrm{Cl})\end{array}$ \\
\hline \multicolumn{4}{|l|}{ Diagnosis } \\
\hline Epicondylitis & $2.0(0.8-5.2)$ & $0.9(0.4-2.2)$ & $1.6(0.5-4.7)$ \\
\hline Impingement, biceps tendonitis, frozen shoulder & $9.6(4.9-18.7)$ & $0.5(0.3-0.8)$ & $2.0(0.9-4.6)$ \\
\hline Other specific diagnosis & $3.7(1.4-9.7)$ & $0.3(0.2-0.5)$ & $5.0(2.2-11.3)$ \\
\hline Nonspecific diagnosis, ref & 1.0 & 1.0 & 1 \\
\hline \multicolumn{4}{|l|}{ Patient and complaint characteristics } \\
\hline Younger age ( $18-45$ y) vs older (46-64 y) & $0.6(0.4-0.9)$ & - & - \\
\hline \multicolumn{4}{|l|}{ Duration of the complaint at consultation: } \\
\hline $0-6$ wk (ref) & 1.0 & 1.0 & 1 \\
\hline 6 wk -6 mo & $1.3(0.8-2.1)$ & $1.7(0.9-3.1)$ & $4.9(1.2-20.4)$ \\
\hline$>6 \mathrm{mo}$ & $1.7(1.0-2.8)$ & $2.4(1.4-4.3)$ & $16.4(4.9-54.8)$ \\
\hline High complaint severity in the last week (score $>6$ ) vs low ${ }^{a}$ & $1.8(1.1-2.9)$ & - & - \\
\hline Many functional limitations (DASH score $>35.34$ ) vs little ${ }^{b}$ & $1.6(1.1-2.4)$ & - & $2.1(1.1-4.1)$ \\
\hline Recurrent complaint vs incident complaint & - & $1.6(1.2-2.1)$ & - \\
\hline Distress, 4DSQc & - & & - \\
\hline Low (score 0-10) & - & 1.0 & - \\
\hline Medium (score 11-20) & - & $1.4(1.0-2.0)$ & - \\
\hline High (score 21-32) & - & $1.9(1.3-2.8)$ & - \\
\hline \multicolumn{4}{|l|}{ GP characteristics } \\
\hline Few years of practice ( $<13$ years) vs many & - & - & $2.3(1.2-4.6)$ \\
\hline Special interest in musculoskeletal complaints vs no & $4.4(1.7-11.4)$ & - & - \\
\hline Rural vs urband & - & $1.9(1.2-3.1)$ & - \\
\hline \multicolumn{4}{|c|}{ All variables in the final model have a multivariate association with a $P<.05$. } \\
\hline \multicolumn{4}{|c|}{$\begin{array}{l}\text { DASH = Disability of Arm Shoulder and Hand questionnaire; } 4 \mathrm{DSQ}=\text { Four-Dimensional Symptom Questionnaire; } O R=\text { odds ratio; } n=\text { number of patients; ref }=\text { refer- } \\
\text { ence group. }\end{array}$} \\
\hline \multicolumn{4}{|c|}{$\begin{array}{l}\text { a Scored on a range from } 1 \text { to } 10 \text { with } 10 \text { being the most severe, and } 6 \text { is the median. } \\
\text { b Each item scored on a } 5 \text {-point Likert scale and summed and transferred to a score ranging from } 0 \text { (no disability) to } 100 \text { (completely disabled). }{ }^{15} \\
\text { c Scored on a range from } 0 \text { to } 32 \text {; a higher score indicates more somatization or distress. }{ }^{19} \\
\text { d Urbanization rate: rural = addresses } \geq 1,000 / \mathrm{km}^{2} \text {, urban }=\text { addresses }>1,000 / \mathrm{km}^{2} .{ }^{20}\end{array}$} \\
\hline
\end{tabular}

and in more confidence in and more frequent use of corticosteroids.

In a study on confidence in primary care management, Glazier et al showed that GPs reported low scores on performing a joint injection or aspiration ${ }^{24}$; the authors proposed that in addition to lack of interest, lack of training and experience is likely to explain the lower scores on confidence. On the other hand, the evidence related to corticosteroid injections was mainly restricted to short-term relief of symptoms, ${ }^{25-28}$ which may be why some GPs selected this option less frequently.

\section{Influence of Diagnosis}

In the subgroups of shoulder complaints and epicondylitis, the Dutch College of General Practitioners has issued management guidelines. ${ }^{6,7}$ The recommended management for shoulder complaints consists of a stepwise approach: watchful waiting (information and wait-and-see), analgesics (ideally, paracetamol; nonsteroidal anti-inflammatories as second-line choice, taken intermittently if no contraindications exist), corticosteroid injections, and referral for exercise therapy if functional limitations are still present after 6 weeks.

For epicondylitis a similar approach is recommended: watchful waiting, analgesics, or corticosteroid injections if pain hinders function. The initial management, as shown in Table 2, seems mainly in line with the guidelines, taking into account the effect of hindrance and duration of the complaint. One difference seems to be the referral for physiotherapy in patients with epicondylitis, an option not recommended in the guideline. ${ }^{6,7}$

\section{Influence of Patient and Complaint Characteristics}

The role complaint variables have in management indicates that, when a patient reports considerable hindrance, the GP will more frequently consider an active treatment option. Additionally, the results show that although medication is prescribed frequently, when patients have a long duration of nontraumatic 


\begin{tabular}{|c|c|c|c|c|c|c|}
\hline Variables & wW & ADT & PM & SI & PT & MS \\
\hline \multicolumn{7}{|l|}{ Diagnosis compared to nonspecific diagnosis } \\
\hline Epicondylitis & $=$ & + & $=$ & $=$ & $=$ & $=$ \\
\hline Impingement, biceps tendonitis, frozen shoulder & $=$ & $=$ & + & + & - & $=$ \\
\hline Other specific diagnosis & $=$ & $=$ & $=$ & + & - & + \\
\hline \multicolumn{7}{|l|}{ Patient and complaint characteristics } \\
\hline Younger (18-45 y) vs older (46-64 y) & $\bullet$ & • & - & - & $\bullet$ & - \\
\hline \multicolumn{7}{|l|}{ Duration of the complaint: compared with $0-6$ wk } \\
\hline 6 wk-6 mo & - & $=$ & - & $=$ & $=$ & + \\
\hline$>6 \mathrm{mo}$ & - & + & - & + & + & + \\
\hline $\begin{array}{l}\text { High complaint severity in the last week (score }>6 \text { ) } \\
\text { vs low }{ }^{\mathrm{a}}\end{array}$ & - & $\bullet$ & $\bullet$ & + & $\bullet$ & $\bullet$ \\
\hline $\begin{array}{l}\text { Many functional limitations (DASH score > } 35.34 \text { ) } \\
\text { vs few }{ }^{\mathrm{b}}\end{array}$ & - & $\bullet$ & + & + & $\bullet$ & + \\
\hline Recurrent complaint vs incident complaint & - & $\bullet$ & • & $\bullet$ & + & $\bullet$ \\
\hline Multiple-region complaint vs single regionc & - & + & • & • & $\bullet$ & $\bullet$ \\
\hline \multicolumn{7}{|l|}{$\begin{array}{l}\text { Somatization, 4DSQ: compared with low } \\
{\text { (score } 0-10)^{d}}^{d}\end{array}$} \\
\hline Medium (11-20) & $\bullet$ & • & $=$ & $\bullet$ & $\bullet$ & $\bullet$ \\
\hline High (21-32) & • & • & + & $\cdot$ & - & • \\
\hline \multicolumn{7}{|l|}{ Distress, 4DSQ: compared with low (score 0-10) } \\
\hline Medium (11-20) & $\bullet$ & • & • & $\bullet$ & + & • \\
\hline High (21-32) & - & • & - & $\bullet$ & + & • \\
\hline \multicolumn{7}{|l|}{ GP characteristics } \\
\hline Female vs male & • & • & - & $\cdot$ & • & • \\
\hline Few years of practice $(<13$ y) vs many & - & + & • & $\bullet$ & $\bullet$ & + \\
\hline Special interest in musculoskeletal complaints vs no & • & • & • & + & $\bullet$ & - \\
\hline Group practice vs solo practice & • & - & - & $\bullet$ & $\bullet$ & • \\
\hline Rural vs urbane & $\bullet$ & • & - & $\bullet$ & + & • \\
\hline \multicolumn{7}{|c|}{$\begin{array}{l}\text { Note: The associations of variables with the } 6 \text { management options are as follows: }+ \text { represents a positive } \\
\text { association; - represents a negative association; }=\text { represents an association similar to the reference category; } \\
\text { represents a variable not associated with a management option. }\end{array}$} \\
\hline \multicolumn{7}{|c|}{$\begin{array}{l}\mathrm{ADT}=\text { additional diagnostic tests; } \mathrm{DASH}=\text { Disability of Arm Shoulder and Hand questionnaire; } 4 \mathrm{DSQ}=\text { Four- } \\
\text { Dimensional Symptom Questionnaire; } \mathrm{GP}=\text { general practitioner; } \mathrm{MS}=\text { medical specialist; } \mathrm{PM}=\text { prescribe a medi- } \\
\text { cation; } \mathrm{PT}=\text { physical therapist; } \mathrm{SI}=\text { steroid injection; } \mathrm{WW}=\text { watchful waiting. }\end{array}$} \\
\hline \multicolumn{7}{|c|}{$\begin{array}{l}\text { a Scored on a range from 1-10 with } 10 \text { being the most severe, and } 6 \text { is the median. } \\
\text { b Each item scored on a } 5 \text {-point Likert scale and summed and transferred to a score ranging from } 0 \text { (no disability) } \\
\text { to } 100 \text { (completely disabled). } .^{15}\end{array}$} \\
\hline \multicolumn{7}{|c|}{$\begin{array}{l}\text { 'Area with the most pain or complaints during the previous week indicated area on a mannequin. Defined } \\
\text { regions are neck-shoulder, including neck; upper part of thoracic spine, shoulder and upper arm; elbow-forearm; } \\
\text { and wrist-hand. }{ }^{7} \\
\text { d Scored on a range from } 0-32 \text {; a higher score indicates more somatization or distress. }{ }^{19} \\
\text { ' Urbanization rate: rural = addresses } \leq 1,000 / \mathrm{km}^{2} \text {, urban }=\text { addresses }>1,000 / \mathrm{km}^{2} .{ }^{20}\end{array}$} \\
\hline
\end{tabular}

located at wrist and hand or fingers were most frequently referred to secondary care, with the highest referral rate for carpal tunnel syndrome. ${ }^{29}$ For patients in this relatively small group whose complaints were of long duration and who had high functional limitations, special treatment or confirmation from a medical specialist seems to have been preferred. Additionally, high somatization scores were positively associated with prescription of medication. An explanation for this association may be that these bodily symptoms are interpreted as being more of a hindrance. Furthermore, in contrast to our expectation, being employed was not an indicator of treatment management.

\section{Influence of Prognosis}

We expected indicators of poor prognosis also to be indicators of management. The associations of long duration of complaints, having a recurrent complaint, and a high somatization score were in line with our expectation. Little social support and having musculoskeletal comorbidity, however, were not associated with any of the 6 management options.

Perhaps the GP's prognosis directly influences management rather than the included indicators of poor prognosis; however, there may be some

arm, neck, or shoulder complaints, other options are explored. ${ }^{6,7}$

No guidelines are available for the largest subgroup of nonspecific complaints. Patients with these complaints are relatively frequently referred for physiotherapy, whereas patients in the small subgroup of having other specific diagnosis are relatively often referred to a medical specialist. Although we report only on initial treatment decisions, these findings seem to be in line with the findings of the 2nd Dutch National Survey of General Practice. There, patients with International Classification of Primary Care codes that included complaints overlap. Because we had collected the 6-month prognosis according to the GP, we also explored its univariate relationship with the 6 management options. Poor prognosis showed a negative association with watchful waiting $(\mathrm{OR}=0.6 ; 95 \% \mathrm{CI}, 0.5-0.9)$. Poor prognosis showed a positive association with additional diagnostic tests $(\mathrm{OR}=2.7 ; 95 \% \mathrm{CI}, 1.7-4.6)$ and with referral for physiotherapy $(\mathrm{OR}=2.1 ; 95 \% \mathrm{CI}, 1.5-3.0)$. The association with referral for medical specialist care $(\mathrm{OR}=1.6 ; 95 \% \mathrm{CI}, 0.7-3.5)$ was not significant (besides the low OR, the prevalence of the outcome was also low). Prescription of medication $(\mathrm{OR}=1.1 ; 95 \% \mathrm{CI}$, 
0.9-1.5) and application of corticosteroid injection $(\mathrm{OR}=0.9 ; 95 \% \mathrm{CI}, 0.6-1.1)$, however, did not show a relation with the expected prognosis. This is in line with the short-term relief of symptoms as a treatment goal with these 2 options while waiting for a positive natural course.

\section{Treatment Options and Evidence}

Although there is evidence of effect for some of the treatment options in nontraumatic arm, neck, and shoulder complaints, these effects are mainly reported as a short-term effect (mainly short-term pain relief) or a limited effect. ${ }^{25-28,30,31}$ The lack of solid evidence in favor of the treatment options explored in our population may explain variability in management and may leave more room for the personal preferences of both the GP and the patient. Aiming at short-term relief of symptoms may lead to favoring a corticosteroid injection, whereas contraindications or fear of side effects of nonsteroidal anti-inflammatory medications may lead to favoring any alternative.

\section{Strengths and Limitations of the Study}

In the present study we studied only initial management in nontraumatic complaints of arm, neck, and shoulder $r_{i}$ thus, additional management options may be applied if the complaints persist. Also, because of the 8 -week response time, $21 \%$ of the patients consulted their GP more than once before filling in the study questionnaire. The number of consultations variable that we added to the final model was associated with all 6 outcomes. Thus, the more consultations, the more frequently a management option other than watchful waiting was applied. Adding the number of consultations to the models hardly changed the odds ratios of the other variables, however. Furthermore, because the different management options were not mutually exclusive, we also checked for co-occurrence of management options. In the initial phase of management, multiple options were applied in $114(17 \%)$ of the patients, mainly analgesics and another option $(\mathrm{n}=94$; 14\%). The most frequent combination was with referral for physiotherapy $(\mathrm{n}=57 ; 8 \%)$. Because our heterogeneous population mainly consists of patients with complaints at neck, shoulder, or elbow, these results mainly apply to these particular subgroups. For patients with certain specific diagnoses (eg, carpal tunnel syndrome; osteoarthritis of elbow, wrist, and/or hand; or hernia of the neck), these results need to be interpreted carefully because of the small numbers. The number of GPs included in the study (31) is not large, but it seems sufficient based on the confidence intervals of the estimates of the GP characteristics.

In the present study we focused on the diagnosis, the patient and complaint, and the GP characteristics and their association with management options. Other variables may also play a role, however. In a prescriptive model for evidence-based clinical decisions, Haynes et al describe a combination of patients' circumstances, patients' preferences and actions, and best evidence research, with an overlap area representing clinical expertise. ${ }^{32}$ Although we included many variables, we did not include patient preference. Furthermore, we did not include such economic variables as insurance and costs, which may also contribute to management choices. ${ }^{33} \mathrm{~A}$ study on medical practice variation in general practice, however, concluded that a clinical rather than an economic model is a more plausible explanation of interpractitioner variation. ${ }^{34}$

\section{Consequences for Practice and Future}

Overall, the diagnostic categories of having a long duration of complaints and reporting many functional limitations were most frequently associated with the choice for a management option. In watchful waiting, the complaint variables (long duration of complaints, high complaint severity, many functional limitations, and a recurrent complaint) played a role in deciding for this management option less frequently. When deciding for 1 of the 5 other management options, however, several GP characteristics played a role as well. That less experience was related to more additional diagnostic tests and more referral for medial specialist care may imply uncertainty regarding the diagnosis. Regarding treatment options, lack of solid evidence in favor of 1 certain option seems to leave room for personal preferences. Further evidence on the effectiveness of management (especially in the large group of nonspecific diagnoses) may reduce variability resulting from GP characteristics.

To read or post commentaries in response to this article, see it online at http://www.annfammed.org/cgi/content/full/7/5/446.

Key words: Primary health care; upper extremity; arm; neck; shoulder; patient care management

Submitted March 8, 2008; submitted, revised, October 31, 2008; accepted December 1, 2008.

Funding support: The study was funded by internal funding of Erasmus MC (Revolving Fund).

Disclaimer: Erasmus MC (Revolving Fund) had no role in the study design, data collection, analysis, interpretation of results, writing the report or in submitting the paper.

Acknowledgments: The authors thank the participating GPs and all the patients for their invaluable contribution to this study. B.W. Koes and S.M.A. Bierma-Zeinstra designed the study. S.M.A. Bierma-Zeinstra was the principal investigator of the study and contributed to the inter- 
pretation of the results and writing of the article. A. Feleus compiled the measuring index, collected the data, executed the analyses, and wrote the article. She had full access to all the study data, and had final responsibility for the decision to submit for publication. R.M.D. Bernsen is a statistician and contributed to the analyses, the interpretation of the results, and writing the article. J.A.N. Verhaar and H.S. Miedema are experts in the field of musculoskeletal complaints and participated in writing the article. B.W. Koes is an expert in epidemiology and contributed to the interpretation of the results, and writing the article.

\section{References}

1. Picavet HS, Schouten JS. Musculoskeletal pain in the Netherlands: prevalences, consequences and risk groups, the DMC(3)-study. Pain 2003:102(1-2):167-178.

2. Walker-Bone K, Palmer KT, Reading I, Coggon D, Cooper C. Prevalence and impact of musculoskeletal disorders of the upper limb in the general population. Arthritis Rheum. 2004;51(4):642-651.

3. Hagen KB, Bjørndal A, Uhlig T, Kvien TK. A population study of factors associated with general practitioner consultation for non-inflam matory musculoskeletal pain. Ann Rheum Dis. 2000;59(10):788-793.

4. Feleus A, Bierma-Zeinstra SMA, Miedema HS, Bernsen RMD, Verhaar JAN, Koes BW. Incidence of non-traumatic complaints of arm, neck or shoulder in general practice. Man Ther. 2008;13(5):426-433.

5. Feleus A, Bierma-Zeinstra SMA, Miedema HS, Verhaar JAN, Koes BW. Management in non-traumatic arm, neck and shoulder complaints: differences between diagnostic groups. Eur Spine J. 2008;17(9):1218-1229.

6. Assendelft WJJ, Rikken SAJJ, Mel M, et al. NHG Standaard Epicondylitis [Dutch College of General Practitioners-practice guideline for epicondylitis], NHG, Utrecht 1997. http://nhgartsennetnl/ upload/104/standaarden/M60/starthtm. Accessed Jan 20, 2006.

7. Winters JC, de Jongh AC, van der Windt DAWM, et al. NHG Standaard Schouderklachten [Dutch College of General Practitionerspractice guideline for shoulder complaints], NHG, Utrecht 1999. http://nhg.artsennet.nl/upload/104/guidelines2/E08.htm. Accessed Jan 20, 2006.

8. Jørgensen $C K$, Fink $P$, Olesen $F$. Patients in general practice in Denmark referred to physiotherapists: a description of patient characteristics based on general health status, diagnoses, and sociodemographic characteristics. Phys Ther. 2001;81(3):915-923.

9. Mäntyselkä P, Ahonen R, Viinamäki H, Takala J, Kumpusalo E. Drug use by patients visiting primary care physicians due to nonacute musculoskeletal pain. Eur J Pharm Sci. 2002;17(4-5):201-206.

10. Côté $P$, Cassidy JD, Carroll L. The treatment of neck and low back pain: who seeks care? Who goes where? Med Care. 2001;39(9):956-967.

11. Feleus A, Bierma-Zeinstra SMA, Miedema HS, et al. Prognostic indicators for non-recovery of non-traumatic complaints at arm, neck and shoulder in general practice-6 months follow-up. Rheumatology (Oxford). 2007;46(1):169-176.

12. Davis PB, Yee RL. Patterns of care and professional decision making in a New Zealand general practice sample. N Z Med J. 1990;103(893):309-312.

13. Cowen ME, Zodet MW. Methods for analyzing referral patterns. J Gen Intern Med. 1999;14(8):474-480.

14. Huisstede BMA, Miedema HS, Verhagen AP, Koes BW, Verhaar JA. Multidisciplinary consensus on the terminology and classification of complaints of the arm, neck and/or shoulder. Occup Environ Med. 2007;64(5):313-319.
15. Hudak PL, Amadio PC, Bombardier C; The Upper Extremity Collaborative Group (UECG). Development of an upper extremity outcome measure: the DASH (disabilities of the arm, shoulder and hand) [corrected]. Am J Ind Med. 1996;29(6):602-608.

16. Ware J Jr, Kosinski M, Keller SD. A 12-Item Short-Form Health Survey: construction of scales and preliminary tests of reliability and validity. Med Care. 1996;34(3):220-233.

17. Von Korff M, Jensen MP, Karoly P. Assessing global pain severity by self-report in clinical and health services research. Spine. 2000;25(24):3140-3151.

18. Feij JA, Doorn CD, van Kampen D, van den Berg PT, Resing WCM. Sensation seeking and social support as moderators of the relationship between life events and physical illness/psyhological distress. In: Winnubst JAM, Maes S, eds. Lifestyles Stress and Health. Leiden: DSWO Press; 1992:285-302.

19. Terluin B, van Marwijk HW, Adèr HJ, et al. The Four-Dimensional Symptom Questionnaire (4DSQ): a validation study of a multidimensional self-report questionnaire to assess distress, depression, anxiety and somatization. BMC Psychiatry. 2006;6:34

20. CBS. http://statline.cbs.nl/statweb/. Accessed Sep 20, 2006.

21. Liang K-Y, Zeger S. Longitudinal data analysis using generalized linear models. Biometrika. 1986;73(1):13-22.

22. Digge PJ, Liang K-Y, Zeger SL. Analysis of Longitudinal Data. New York, NY: Oxford University Press; 1994.

23. Salem-Schatz S, Moore G, Rucker M, et al. The case for case-mix adjustment in practice profiling. When good apples look bad. JAMA. 1995;273(10):772-773.

24. Glazier RH, Dalby DM, Badley EM, Hawker GA, Bell MJ, Buchbinder R. Determinants of physician confidence in the primary care management of musculoskeletal disorders. J Rheumatol. 1996;23(2):351-356.

25. Kumar A, Djulbegovic B, Struijs P. Myeloma (multiple). Clin Evid. 2006;15(15):1-29.

26. Kumar A, Djulbegovic B. Myeloma (multiple). Clin Evid. 2006; 15:1-29.

27. Ashworth N. Carpal tunnel syndrome. BMJ. Clin Evid. 2006;15:1-3.

28. Marshall S, Tardif G, Asworth N. Local cortocsteroid injection for carpal tunnel syndrome. (Cochrane Review). Cochrane Database Syst Rev. 2007;(2):CD001554.

29. Cardol M, van Dijk L, de Jong JD, de Bakker DH, Westert GP. De tweede Nationale Studie naar ziekten en verrichtingen in de huisartsenpraktijk: Huisartsenzorg: wat doet de poortwachter [Dutch]. Utrecht/Bilthoven: Nivel/RIVM 2004

30. van Tulder M, Malmivaara A, Koes B. Repetitive strain injury. Lancet. 2007;369(9575):1815-1822

31. Verhagen AP, Karels C, Bierma-Zeinstra SMA, et al. Ergonomic and physiotherapeutic interventions for treating work-related complaints of the arm, neck or shoulder in adults. Cochrane Database Syst Rev. 2006;3(Issue 3):CD003471.

32. Haynes RB, Devereaux PJ, Guyatt GH. Physicians' and patients' choices in evidence based practice. BMJ. 2002;324(7350):1350.

33. Freburger JK, Holmes GM, Carey TS. Physician referrals to physical therapy for the treatment of musculoskeletal conditions. Arch Phys Med Rehabil. 2003;84(12):1839-1849.

34. Davis PB, Gribben B, Scott A, Lay-Yee R. The "supply hypothesis" and medical practice variation in primary care: testing economic and clinical models of inter-practitioner variation. Soc Sci Med. 2000:50(3):407-418 